\title{
Theory and Simulation of CSR Microbunching in Bunch Compressors
}

\author{
Zhirong Huanga*, Michael Borland ${ }^{\mathrm{a}}$, Paul Emma ${ }^{\mathrm{b}}$, Kwang-Je Kim ${ }^{\mathrm{a}}$ \\ ${ }^{a}$ Argonne National Laboratory, Argonne, IL 60439, USA \\ ${ }^{\mathrm{b}}$ Stanford Linear Accelerator Center, Stanford, CA 94309, USA
}

CSR microbunching instability in bunch compressors is studied both analytically and numerically. The iterative solutions of the integral equation for the instability provide approximate expressions of CSR microbunching due to initial density and energy modulation, and can be applied to a series of bending systems consisting of multiple compressor chicanes and transport lines. Two similar but independent simulation methods are developed and are compared to each other as well as with theory. We determine the total gain in density modulation for all bend systems of the Linac Coherent Light Source and discuss initial conditions that start the unstable process.

\section{Introduction}

A very challenging requirement for a future $\mathrm{x}-$ ray free-electron laser (FEL) is the generation of a low-emittance $(\gamma \varepsilon \sim 1 \mu \mathrm{m})$, high-current (a few $\mathrm{kA}$ ) electron beam $[1,2]$. The typical accelerator design to reach this goal starts with a photocathode rf gun capable of producing the required emittance at a much lower current and uses a series of magnetic bunch compressors to reduce the bunch length and increase the peak current correspondingly. Coherent synchrotron radiation (CSR) induced by a short bunch in the bends of these compressor chicanes is a major concern because CSR can interact with the bunch and cause an emittance growth [3]. Recently, computer simulations have indicated that small modulations in bunch density can be significantly amplified by its induced CSR field, giving rise to a microbunching instability [4]. Such an instability is the subject of intense theoretical studies [5-7]. In this paper, we discuss the amplification mechanism and present analytic formulas for calculating the amplification factor. Systematic numerical simulations are carried out to study the instability and to compare with the theoretical results. Finally, the analytical and the numerical tools are applied to bend systems of the Linac Coherent Light Source (LCLS) [1], where the effect was first discovered through the tracking study [4].

$\overline{{ }^{*} \text { Corresponding author (Email: zrh@aps.anl.gov) }}$

\section{Theory}

A typical bunch compressor chicane consists of four dipoles of equal length $L_{b}$ and equal magnitude of bending radius $\rho_{0}$. The middle two dipoles are very close to each other while the first and the last dipoles are $\Delta L$ apart from their nearest neighbors. An incoming beam with an energy chirp $h=\Delta \delta /(\Delta z)$ (relative energy change per unit bunch length) will be compressed in the chicane by an amount $\left(1+h R_{56}\right)^{-1}$, where $R_{56} \approx-2 \frac{L_{b}^{2}}{\rho_{0}^{2}}\left(\Delta L+\frac{2}{3} L_{b}\right)<0$ since the head of the bunch is at $z<0$.

Suppose the beam current is modulated at a wavelength $\lambda=2 \pi / k$ that is much smaller than the bunch length. CSR can be emitted for this wavelength at a location $\tau$ in a dipole and induce beam energy modulation at the same wavelength due to the CSR impedance [8]. Through the momentum compaction $R_{56}(\tau \rightarrow s)$, such energy modulation can be turned into density modulation at a later chicane location $s$. The process will repeat at $s$, leading to the amplification of the current modulation, or microbunching. Defining a bunching parameter $b(k ; s)$ as the Fourier transform of the current, the linear evolution of $b(k ; s)$ may be described by an integral equation $[6,7]$. The integral equation assumes a coasting beam (infinite bunch length) but takes into account the smearing effects of the initial beam emittance $\varepsilon_{x}$ and incoherent energy spread $\sigma_{\delta}$. When the drift 
space between dipoles $\Delta L \gg L_{b}$, the induced density modulation from the energy modulation in the same dipole is normally negligible [5], and the integral equation is solved by two iterations to $\mathrm{O}\left(L_{b} / \Delta L\right)$ [7]. For simplicity, we use the steadystate, free-space CSR wake or impedance $[3,8]$. We also assume the twiss parameters $\beta_{0} \gg L_{b}$ and $\left|\alpha_{0}\right| \sim 1$ at the entrance of the chicane. For an initial density modulation characterized by the bunching parameter $b_{0}\left(k_{0} ; 0\right)$, the final bunching at $k_{f}=k_{0} /\left(1+h R_{56}\right)$ is given by [7]

$$
\begin{aligned}
& \frac{b\left(k_{f} ; f\right)}{b_{0}\left(k_{0} ; 0\right)} \approx \exp \left[-\frac{\bar{\sigma}_{\delta}^{2}}{2(1+\chi)^{2}}\right]+A \bar{I}_{f}\left[\frac{\sqrt{\pi} \operatorname{erf}\left(\bar{\sigma}_{x}\right)}{2 \bar{\sigma}_{x}}\right. \\
& \left.\times \exp \left(-\frac{\bar{\sigma}_{\delta}^{2}}{2(1+\chi)^{2}}\right)+F_{1}\left(\chi, \bar{\sigma}_{x}, \alpha_{0}, \phi, \bar{\sigma}_{\delta}\right)\right] \\
& +A^{2} \bar{I}_{f}^{2} F_{0}\left(\bar{\sigma}_{x}\right) F_{2}\left(\chi, \bar{\sigma}_{x}, \alpha_{0}, \phi, \bar{\sigma}_{\delta}\right) .
\end{aligned}
$$

Here $\chi=h R_{56}, \bar{\sigma}_{\delta}=k_{0} R_{56} \sigma_{\delta}, A=1.63 i-$ $0.94, \bar{I}_{f}=I_{f} k_{0}^{4 / 3} R_{56} L_{b} /\left(\gamma I_{A} \rho_{0}^{2 / 3}\right), I_{f}$ is the compressed beam current, $I_{A} \approx 17 \mathrm{kA}, \bar{\sigma}_{x}=$ $k_{0} L_{b} \sqrt{\varepsilon_{x} \beta_{0}} / \rho_{0}, \phi=2 \Delta L / \beta_{0}$, the error function $\operatorname{erf}(x)=2 \pi^{-1 / 2} \int_{0}^{x} d t \exp \left(-t^{2}\right)$, and

$$
\begin{aligned}
F_{0}= & \frac{e^{-\bar{\sigma}_{x}^{2}}+\bar{\sigma}_{x} \sqrt{\pi} \operatorname{erf}\left(\bar{\sigma}_{x}\right)-1}{2 \bar{\sigma}_{x}^{2}}, \\
F_{1}= & 2 \int_{0}^{1} d t \frac{(1-t)}{(1+\chi t)^{4 / 3}} H(t), \\
F_{2}= & 2 \int_{0}^{1} d t \frac{(1-t) t(1+\chi)}{(1+\chi t)^{7 / 3}} H(t), \\
H(t)= & \exp \left[-\bar{\sigma}_{x}^{2} \frac{\left(1-2 t+\alpha_{0} \phi t\right)^{2}+\phi^{2} t^{2}}{(1+\chi t)^{2}}\right. \\
& \left.-\frac{\bar{\sigma}_{\delta}^{2}}{2(1+\chi t)^{2}}\left(t^{2}+\frac{(1-t)^{2}}{(1+\chi)^{2}}\right)\right] .
\end{aligned}
$$

The first term on the right side of Eq. (1) is the bunching degradation in the absence of any CSR; the second term, linear in current, is the result of one-stage amplification and is dominant in lowgain situations; and the last term, quadratic in current, is the result of two-stage amplification and is dominant when the gain is high. In particular, it reduces to Eq. (26) of Ref. [5] in the absence of compression (i.e., $h=0$ ) and when the emittance is zero. Equation (1) also agrees with numerical solutions of the integral equation [6].
CSR microbunching can also be initiated by an energy modulation $p\left(k_{0} ; 0\right)$. It is turned into density modulation through $R_{56}$, which will be further amplified by CSR. The final bunching from the initial energy modulation is [7]

$$
\begin{aligned}
b^{p}\left(k_{f} ; f\right) \approx & -i k_{f} R_{56} p\left(k_{0} ; 0\right)\left[\exp \left(-\frac{\bar{\sigma}_{\delta}^{2}}{2(1+\chi)^{2}}\right)\right. \\
& \left.+A \bar{I}_{f} F_{2}\left(\chi, \bar{\sigma}_{x}, \alpha_{0}, \phi, \bar{\sigma}_{\delta}\right)\right]
\end{aligned}
$$

\section{Simulation}

Two simulation codes (elegant and CSR_calc) are used to study the CSR microbunching. The CSR algorithm in both codes is based on an analytical model for the longitudinal CSR wake [9] that includes both the steady-state $[3,8]$ and transient effects [10]. To reduce the numerical noise, a few million macroparticles are chosen using a quiet-start method based on Halton sequences for all phase-space coordinates. A sinusoidal density modulation is added smoothly to the current profile. The gain in the modulation amplitude after the chicane is examined for various $\lambda$.

For simulations with elegant [11], we use a flat-top current distribution of length $100 \lambda$, with 4- $\sigma$ Gaussian ends having Gaussian parameter $\sigma_{e}=10 \lambda$. The modulation amplitude is varied between $0.0625 \%$ and $4 \%$, as needed for control of noise and saturation effects. A bin size of $\lambda / 20$ was used in the CSR algorithm. The gain was computed by comparing the amplitude of the component at $\lambda$ (or its compressed equivalent), as determined using Laskar's method [11]. CSR_calc uses a similar scheme but a Gaussian current profile with $\pm 3 \sigma_{z}$ cutoff. The modulation on the current profile is reevaluated after the chicane by averaging over $\pm 0.2 \sigma_{z}$ near the peak current.

As a numerical example, we compute the gain for a model chicane (see CSR workshop 2002, http://www.desy.de/csr/). We use a chirped electron beam $\left(h=36 \mathrm{~m}^{-1}\right)$ at $5 \mathrm{GeV}$ with $1-\mu \mathrm{m}$ normalized emittance and $2 \times 10^{-6}\left(2 \times 10^{-5}\right)$ incoherent energy spread initially. The compressed current is assumed to be $6 \mathrm{kA}$. Figure 1 shows simulation results of elegant and CSR_calc us- 


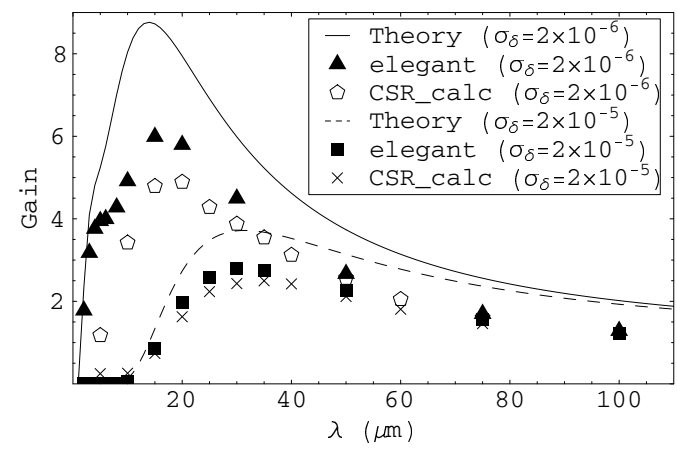

Figure 1. Gain of a model chicane as a function of the initial modulation wavelength.

ing steady-state CSR, in comparison with theory (i.e., Eq. (1)). Tracking with transient CSR gives very similar results. Gain difference of theory, elegant, and CSR_calc may come from the different assumptions in bunch length and shape.

\section{Applications to the LCLS}

The LCLS accelerator consists of four bend systems, two of which are bunch compressor chicanes (BC1 and BC2). To damp the CSR microbunching instability, a superconducting wiggler before $\mathrm{BC} 2$ can be used to increase the incoherent energy spread $\sigma_{\delta}$ at $4.5 \mathrm{GeV}$ (from $3 \times 10^{-6}$ to $3 \times 10^{-5}$ ). Figure 2 shows a schematic of the LCLS layout, and more details can be found in Ref. [1]. In what follows, $\gamma \varepsilon_{x}$ is assumed to be $1 \mu \mathrm{m}$ throughout the accelerator, the final bunch current is $4 \mathrm{kA}$, and $\sigma_{\delta}$ is given at various stages in Fig. 2. For each compressor, the gain in density modulation is found to be less than 3 in theory as well as in simulation $[6,7,12]$. Putting them in series, $\mathrm{BC} 2$ not only amplifies the density modulation gained in $\mathrm{BC} 1$, but also turns the energy modulation accumulated in $\mathrm{BC} 1$ into density modulation and amplifies it subsequently. Thus, the total gain of the two-compressor system is higher than the product of the gains of each compressor and is analyzed in Ref. [7]. We carry out CSR_calc simulations by modeling the linac sections between

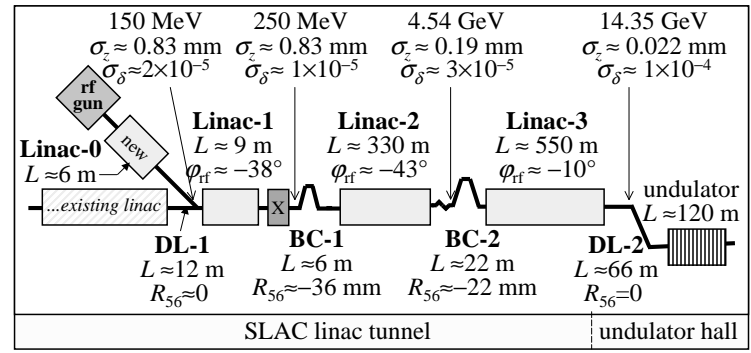

Figure 2. Layout of the LCLS bend systems.

$\mathrm{BC} 1$ and $\mathrm{BC} 2$ with a linear transformation of the beam which produces the proper energy, incoherent energy spread, linear chirp, emittance, and twiss parameters at the entrance to $\mathrm{BC} 2$, while retaining the evolving current and energy modulations. This simplification eliminates the complications induced by nonlinearity and rf structure wakefields and allows a more consistent comparison with theory, which is shown in Fig. 3 .

In addition to $\mathrm{BC} 1$ and $\mathrm{BC} 2$, two other bend systems (the "dog-legs" DL1 and DL2, see Fig. 2) can also contribute to CSR microbunching. These dog-legs are simple transport lines and have much smaller magnitude of $R_{56}$ than the compressors. Thus, we may neglect the induced density modulation in these DLs but keep track of the induced energy modulation in the analytical calculation. We first find the energy modulation induced by an initial density modulation in DL1 using Eq. (39) of Ref. [7]. This density modulation together with the induced energy modulation in DL1 will be amplified first in $\mathrm{BC} 1$ and then in $\mathrm{BC} 2$, as determined by Eqs. (1) and (3). Since DL2 can only induce energy modulation but not density modulation, the total gain in density modulation for the entire LCLS bend system is mainly determined by the first three bend systems DL1, BC1, and $\mathrm{BC} 2$ and is shown in Fig. 4 with and without the effect of the wiggler. Corresponding CSR_calc simulations using the linear transformations between the four bend systems (as described in the previous paragraph) show reasonable agreement with theory (see Fig. 4). Using the full accelerator model including all nonlinearity and wakefield 


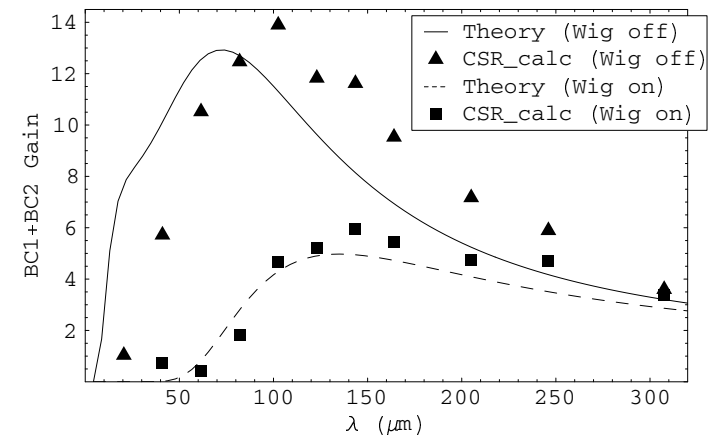

Figure 3. Total gain of the two LCLS compressors as a function of the initial modulation wavelength with and without the damping wiggler.

effects, elegant simulations shown in Fig. 4 indicate more gain than the linear theory and linear simulations. Figures 3 and 4 also illustrate the damping effect of the wiggler.

In the linear regime of the instability (i.e., when $|b| \ll 1)$, both the sliced emittance and the incoherent energy spread are unaffected by the process. However, in the nonlinear regime where $|b|$ approaches 1 , we expect significant increases in both quantities due to the CSR microbunching. For a bunch with a smooth longitudinal profile, the initial density modulation is mainly determined by shot noise fluctuations with $\left|b_{\text {noise }}\right| \sim N_{\text {coh }}^{-1 / 2} \sim 10^{-5}$, where the number of electrons within the coherence length $N_{\text {coh }}$ is comparable to the total number of electrons. With a peak gain of no more than 100 even without the wiggler, $\left|b_{\text {final }}\right| \ll 1$ from the shot noise start-up. Nevertheless, if sharp subbunch structures are produced in a photocathode rf gun, $\left|b_{\text {initial }}\right| \gg\left|b_{\text {noise }}\right|$, and $\left|b_{\text {final }}\right|$ may become close to 1 with a significant gain. Thus, the damping wiggler in the LCLS reduces the gain as well as the sensitivity to the initial bunch shape.

We thank G. Stupakov for many stimulating discussions. This work was supported by the U. S. Department of Energy under Contract Nos. W31-109-ENG-38 and DE-AC03-76SF00515.

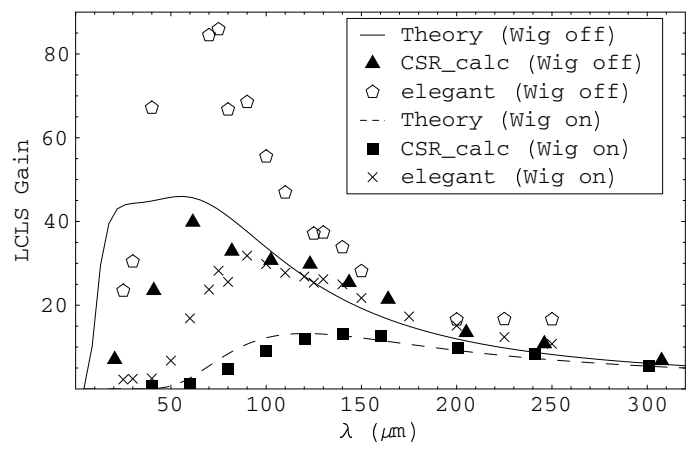

Figure 4. Total gain of the entire LCLS bend system as a function of the initial modulation wavelength with and without the wiggler.

\section{REFERENCES}

1. Linac Coherent Light Source Conceptual Design Report, http://wwwssrl.slac.stanford.edu/lcls/CDR/ (2002).

2. TESLA Techinical Design Report, DESY TESLA-FEL 2001-05 (2001).

3. Y.S. Derbenev et al., DESY TESLA-FEL 9505 (1995).

4. M. Borland et al., in Proc. of PAC 2001, 2707 (IEEE, Piscataway, NJ, 2001); Nucl. Instrum. Methods Phys. Res., Sect. A 483, 268 (2002).

5. E.L. Saldin, E.A. Schneidmiller, M.V. Yurkov, DESY TESLA-FEL-2002-02 (2002).

6. S. Heifets, G. Stupakov, S. Krinsky, Phys. Rev. ST Accel. Beams 5, 064401 (2002).

7. Z. Huang, K.-J. Kim, Phys. Rev. ST Accel. Beams 5, 074401 (2002).

8. J.B. Murphy, S. Krinsky, R.L. Gluckstern, in Proc. of PAC 1995, 2980 (IEEE, Piscataway, NJ, 1995); Particle Accelerators 57, 9 (1997).

9. G. Stupakov, P. Emma, to be published in Proc. of EPAC 2002, Paris, France, 2002.

10. E.L. Saldin et al., Nucl. Instrum. Methods Phys. Res., Sect. A 398, 373 (1997).

11. M. Borland, to be published in Proc. of Linac 2002, Kyongju, Korea, 2002.

12. P. Emma, to be published in Proc. of EPAC 2002, Paris, France, 2002. 